\title{
On the Slope of the Elastic Differential Cross Sections
}

\author{
S. D. Campos, M. J. Menon, and J. Montanha \\ Instituto de Física Gleb Wataghin \\ Universidade Estadual de Campinas, UNICAMP \\ 13083-970 Campinas, SP, Brazil
}

Received on 29 September, 2006

\begin{abstract}
Making use of a model-independent analytical fit for the elastic hadron-hadron amplitude, recently developed, we investigate the slope of the proton-proton and antiproton-proton differential cross sections, as a function of the energy and the momentum transfer. We show that the predictions for these quantities are in agreement with the experimental data available and discuss the effect of the slope position as function of the momentum transfer.

Keywords: Elastic scattering; Hadron-induced high-energy interactions
\end{abstract}

\section{INTRODUCTION}

In the absence of a pure QCD description of high-energy elastic hadron scattering (soft diffractive processes), empirical parametrizations for the scattering amplitude and fits to the experimental data play an important role in the extraction of model-independent information on the scattering process [1]. However, one disadvantage of this kind of analysis is its local character, namely the free parameters are inferred from fits to each energy and interaction process, leading therefore to a nonpredictive approach.

Recently, in [2] it has been developed a model-independent and predictive analysis of elastic scattering at high energies. In this approach, a novel analytical parametrization for the imaginary part of the amplitude is inferred on empirical grounds and based on some rigorous high-energy theorems and bounds from axiomatic quantum field theory. The corresponding real part of the amplitude is analytically evaluated by means of dispersion relations (connecting, therefore, particle-particle and particle-antiparticle scattering). Simultaneous fits to the experimental data on total cross section, $\rho$ parameter and differential cross section, from both protonproton $(p p)$ and antiproton-proton $(\bar{p} p)$ scattering, above 20 $\mathrm{GeV}$, lead to a predictive and model-independent approach, able to describe quite well the bulk of the experimental data. All the details of the analysis can be found in [2].

In this communication we present novel results concerning only the slope of the differential cross section for the above reactions. Specifically, we evaluate this quantity in an analytical way and investigate the effects of the predictions in the experimental data as function of the energy and the momentum transfer. The manuscript is organized as follows. In Sec. 2 we shortly review some main formulas in the analysis and the fit procedure. In Sec. 3 we present the results for the slopes. The conclusions and some final remarks are the contents of Sec. 4.

\section{ANALYTICAL PARAMETRIZATION AND FITTING}

\section{A. Analytical Parametrization}

In [2] it has been analyzed all the experimental data, available above $\sqrt{s}=20 \mathrm{GeV}$, on differential cross sections

$$
\frac{d \sigma}{d q^{2}}=\frac{1}{16 \pi s^{2}}\left|\operatorname{Re} F\left(s, q^{2}\right)+i \operatorname{Im} F\left(s, q^{2}\right)\right|^{2}
$$

total cross section

$$
\sigma_{t o t}(s)=\frac{\operatorname{Im} F\left(s, q^{2}=0\right)}{s},
$$

and the $\rho$ parameter

$$
\rho(s)=\frac{\operatorname{Re} F\left(s, q^{2}=0\right)}{\operatorname{Im} F\left(s, q^{2}=0\right)},
$$

from $p p$ and $\bar{p} p$ scattering. The imaginary parts of the amplitudes are parametrized by

$$
\frac{\operatorname{Im} F_{p p}\left(s, q^{2}\right)}{s}=\sum_{i=1}^{n} \alpha_{i}(s) e^{-\beta_{i}(s) q^{2}}
$$

with

$$
\begin{aligned}
& \alpha_{i}(s)=A_{i}+B_{i} \ln (s)+C_{i} \ln ^{2}(s), \\
& \beta_{i}(s)=D_{i}+E_{i} \ln (s),
\end{aligned}
$$

and

$$
\frac{\operatorname{Im} F_{\bar{p} p}\left(s, q^{2}\right)}{s}=\sum_{i=1}^{n} \bar{\alpha}_{i}(s) e^{-\bar{\beta}_{i}(s) q^{2}}
$$

with

$$
\begin{aligned}
\bar{\alpha}_{i}(s) & =\bar{A}_{i}+\bar{B}_{i} \ln (s)+\bar{C}_{i} \ln ^{2}(s), \\
\bar{\beta}_{i}(s) & =\bar{D}_{i}+\bar{E}_{i} \ln (s),
\end{aligned}
$$

where $A_{i}, B_{i}, \ldots E_{i}, \bar{A}_{i}, \bar{B}_{i}, \ldots \bar{E}_{i}$ are real constants (parameters to be fitted) and $i=1,2, \ldots n$. In order that the above parametrizations might be in agreement with rigorous results from 
the axiomatic quantum field theory, the following constraint has been also used

$$
\sum_{i=1}^{n}\left(C_{i}-\bar{C}_{i}\right)=0 .
$$

The corresponding real parts of the amplitudes are evaluated by means of derivative dispersion relations (see [3] for a recent review), extended beyond the forward direction. These relations are expressed in terms of even $(+)$ and odd $(-)$ amplitudes and the connections with the hadronic amplitudes are established through the usual definitions $F_{p p}\left(s, q^{2}\right)=$ $F_{+}\left(s, q^{2}\right)+F_{-}\left(s, q^{2}\right)$ and $F_{\bar{p} p}\left(s, q^{2}\right)=F_{+}\left(s, q^{2}\right)-F_{-}\left(s, q^{2}\right)$, leading to analytical expressions for the real parts (see [2] for the explicit equations). With this formalism the physical quantities $(1-3)$ are also analytically obtained.

\section{B. Fitting and Data}

For the forward data on $\sigma_{t o t}$ and $\rho$, it has been used the Particle Data Group archives. The differential cross section data concern the optical point and the data above the Coulombnuclear interference region. The ensemble includes 12 data sets in the region $0.01<q^{2}<14 \mathrm{GeV}^{2}: p p$ scattering at $\sqrt{s}=$ $23.5,27.4,30.7,44.7,52.8$, and $62.5 \mathrm{GeV}$ and $\bar{p} p$ scattering at $\sqrt{s}=31,53,61,546,630$ and $1800 \mathrm{GeV}$ (complete list of references to this ensemble can be found in [1].)

Simultaneous fits have been performed to $\sigma_{t o t}(s), \rho(s)$ and $d \sigma\left(s, q^{2}\right) / d q^{2}$ data, from $p p$ and $\bar{p} p$ scattering, through the CERN-Minuit code. As discussed in detail in [2], we have considered four cutoffs for the maximum value of the momentum transfer in the differential cross section, namely data up to $q_{\max }^{2}=1.0,1.5,2.0$ and $14.0 \mathrm{GeV}^{2}$ (all the differential cross section data included). In this communication we shall use only the results with $q_{\max }^{2}$ up to $2 \mathrm{GeV}^{2}$ and $14 \mathrm{GeV}^{2}$. As said before all the strategies, details and extensive discussion on these and other results can be found in [2]. In what follows we shall only use these results in the prediction of the slopes of the $p p$ and $\bar{p} p$ differential cross section data, without free parameter.

\section{RESULTS FOR THE SLOPES}

The slope is defined by

$$
B\left(s, q^{2}\right)=-\frac{d}{d q^{2}}\left[\ln \frac{d \sigma}{d q^{2}}\left(s, q^{2}\right)\right]
$$

where $q^{2}=-t$. From Eq. (1) and parametrizations (4 - 9) this quantity is analytically evaluated:

$$
\begin{aligned}
& B_{p p}\left(s, q^{2}\right)=\frac{-2\left(\Delta_{1} \Delta_{2}+\Delta_{3}\right)}{\Delta_{1}^{2}+\Delta_{4}^{2}}, \\
& B_{\bar{p} p}\left(s, q^{2}\right)=\frac{-2\left(\bar{\Delta}_{1} \bar{\Delta}_{2}+\bar{\Delta}_{3}\right)}{\bar{\Delta}_{1}^{2}+\bar{\Delta}_{4}^{2}},
\end{aligned}
$$

where $\Delta_{i}$ and $\bar{\Delta}_{i}, i=1,2,3,4$ are functions of $s$ and $q^{2}$, given, explicitly, by

$$
\begin{gathered}
\Delta_{1}=\frac{k}{s}+\sum_{i=1}^{n}\left\{\frac{\pi}{2}\left[\alpha_{i}^{\prime}(s)-\alpha_{i}(s) \beta_{i}^{\prime}(s) q^{2}\right] e^{-\beta_{i}(s) q^{2}}+\frac{\pi}{4}\left[\alpha_{i}(s) e^{-\beta_{i}(s) q^{2}}-\bar{\alpha}_{i}(s) e^{-\beta_{i}(s) q^{2}}\right]\right\} \\
\Delta_{2}=-\sum_{i=1}^{n}\left\{\frac{\pi}{2}\left[\alpha_{i}^{\prime}(s) \beta_{i}(s)+\alpha_{i}(s) \beta_{i}^{\prime}(s)-\alpha_{i}(s) \beta_{i}(s) \beta_{i}^{\prime}(s) q^{2}\right] e^{-\beta_{i}(s) q^{2}}+\frac{\pi}{4}\left[\alpha_{i}(s) \beta_{i}(s) e^{-\beta_{i}(s) q^{2}}-\bar{\alpha}_{i}(s) \bar{\beta}_{i}(s) e^{\left.\left.-\bar{\beta}_{i}(s) q^{2}\right]\right\}}\right.\right. \\
\Delta_{3}=-\sum_{i=1}^{n} \alpha_{i}(s) e^{-\beta_{i}(s) q^{2}} \sum_{i=1}^{n} \alpha_{i}(s) \beta_{i}(s) e^{-\beta_{i}(s) q^{2}}, \quad \Delta_{4}=\sum_{i=1}^{n} \alpha_{i}(s) e^{-\beta_{i}(s) q^{2}} \\
\bar{\Delta}_{1}=\frac{k}{s}+\sum_{i=1}^{n}\left\{\frac{\pi}{2}\left[\bar{\alpha}_{i}^{\prime}(s)-\bar{\alpha}_{i}(s) \bar{\beta}_{i}^{\prime}(s) q^{2}\right] e^{-\bar{\beta}_{i}(s) q^{2}}+\frac{\pi}{4}\left[\alpha_{i}(s) e^{-\beta_{i}(s) q^{2}}-\bar{\alpha}_{i}(s) e^{-\beta_{i}(s) q^{2}}\right]\right\} \\
\bar{\Delta}_{2}=-\sum_{i=1}^{n}\left\{\frac{\pi}{2}\left[\bar{\alpha}_{i}^{\prime}(s) \bar{\beta}_{i}(s)+\bar{\alpha}_{i}(s) \bar{\beta}_{i}^{\prime}(s)-\bar{\alpha}_{i}(s) \bar{\beta}_{i}(s) \bar{\beta}_{i}^{\prime}(s) q^{2}\right] e^{-\bar{\beta}_{i}(s) q^{2}}+\frac{\pi}{4}\left[\alpha_{i}(s) \beta_{i}(s) e^{-\beta_{i}(s) q^{2}}-\bar{\alpha}_{i}(s) \bar{\beta}_{i}(s) e^{-\bar{\beta}_{i}(s) q^{2}}\right]\right\} \\
\bar{\Delta}_{3}=-\sum_{i=1}^{n} \bar{\alpha}_{i}(s) e^{-\bar{\beta}_{i}(s) q^{2}} \sum_{i=1}^{n} \bar{\alpha}_{i}(s) \bar{\beta}_{i}(s) e^{-\bar{\beta}_{i}(s) q^{2}}, \quad \bar{\Delta}_{4}=\sum_{i=1}^{n} \bar{\alpha}_{i}(s) e^{-\bar{\beta}_{i}(s) q^{2}}
\end{gathered}
$$

Making use of the free parameters given in [2] we can, therefore, evaluate $B$ as function of $s$ and $q^{2}$ for $p p$ and $\bar{p} p$ scattering. As stated before, among the fits discussed in this 

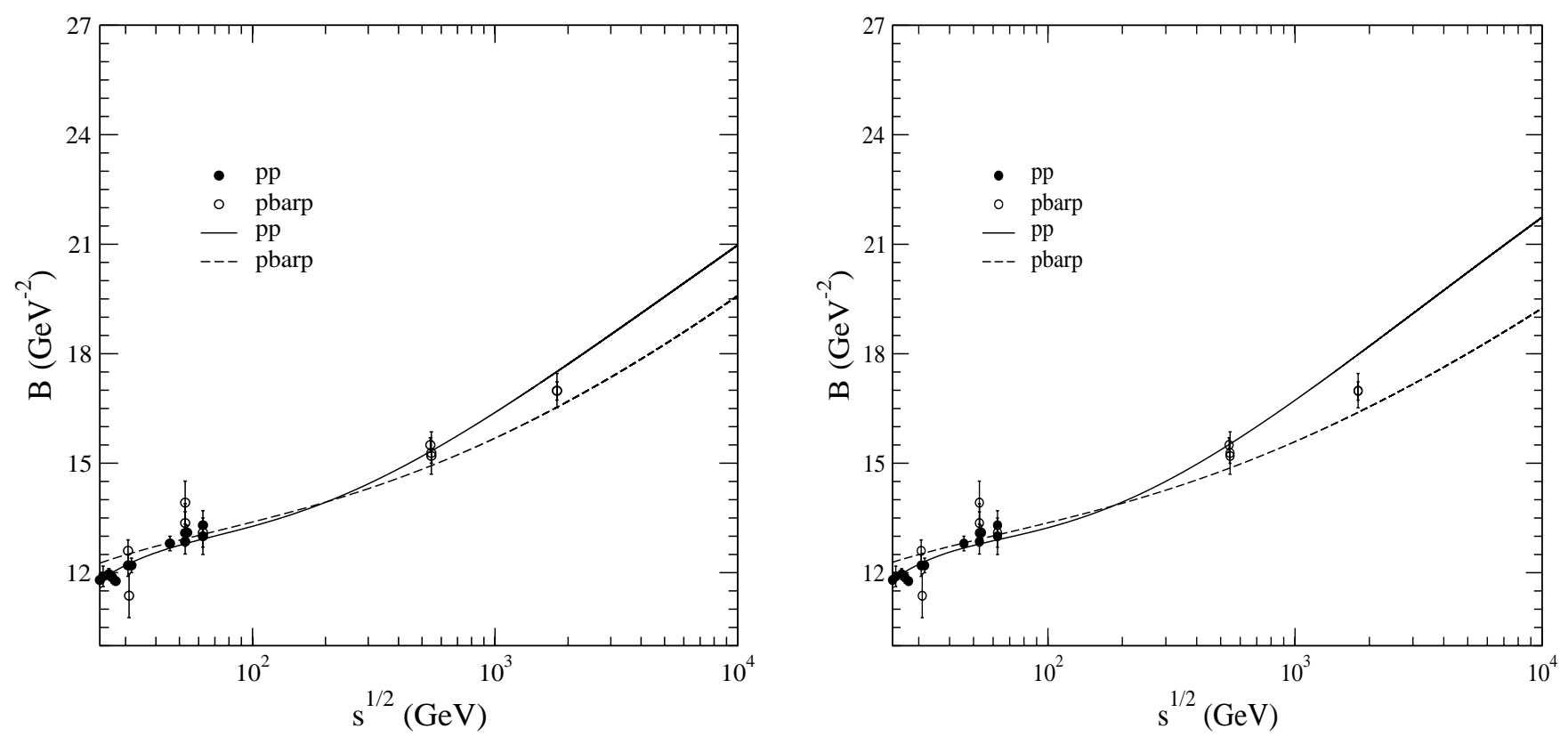

FIG. 1: Predictions for the slope at $q^{2}=0.05 \mathrm{GeV}^{2}$ as function of the energy, from fits to the differential cross section data up to $q_{\mathrm{max}}^{2}=2$ $\mathrm{GeV}^{2}$ (left) and $q_{\max }^{2}=14 \mathrm{GeV}^{2}$ (right).

reference, we shall consider here only those with differential cross section data up to $q_{\max }^{2}=2 \mathrm{GeV}^{2}$ and $q_{\max }^{2}=14 \mathrm{GeV}^{2}$. These cases can give information on the influence of the differential cross section on the global predictions, as discussed in what follows.

The main point is to compare the predictions for the slope, as function of the energy, with the experimental data on $p p$ and $\bar{p} p$ scattering. However, since this quantity also depends on the momentum transfer and the experimental data are usually obtained in the interval $q^{2} \approx 0-0.1 \mathrm{GeV}^{2}$, we shall evaluate the predictions at a roughly average point situated at $q^{2}=0.05 \mathrm{GeV}^{2}$.

The results from the fits to total cross section, $\rho$ and differential cross section up to $q_{\max }^{2}=2 \mathrm{GeV}^{2}$ and $q_{\max }^{2}=14 \mathrm{GeV}^{2}$ [2] are displayed in Fig. 1 together with the experimental data [4]. We see that the results show an agreement with the experimental data and predict a faster increase of the slope for the $p p$ scattering than for the $\bar{p} p$ at the highest energies. The effect of the data at high momentum transfer $\left(q_{\max }^{2}=14 \mathrm{GeV}^{2}\right)$ indicate a faster increase in this difference.

Since, in general, model predictions for the slope present only the forward value, namely $B$ at $q^{2}=0$, we also evaluate $B\left(s, q^{2}=0\right)$ as a complementary test. The results are shown in Fig. 2, together with the experimental data and also for the two cases: $q_{\max }^{2}=2 \mathrm{GeV}^{2}$ and $q_{\max }^{2}=14 \mathrm{GeV}^{2}$. We see that although the descriptions of the data are similar to the results in Fig. 1, the predictions at the highest energies indicate a faster increase of the slopes and also in the differences between $p p$ and $\bar{p} p$ scattering.

\section{CONCLUSIONS}

Making use of a model independent and predictive formalism, based on the analysis of $p p$ and $\bar{p} p$ differential cross sections, total cross sections and $\rho$ parameter, we presented here the predictions for the slope of the differential cross section. Since the slope data did not take part in the original fits, the comparison of the predictions with the experimental data represent a good test for the formalism.

We considered four variants: results of the original fits with differential cross section data up to $q_{\max }^{2}=2 \mathrm{GeV}^{2}$ and $q_{\max }^{2}=$ $14 \mathrm{GeV}^{2}$ and in each case, the evaluation of the slope at $q^{2}=$ $0.05 \mathrm{GeV}^{2}$ and $q^{2}=0$. With this strategy we tested not only the influence of the differential cross section data, but also the influence of the momentum transfer on the calculated values of the local slopes.

From Figs. 1 and 2 we see that the predictions in all the four variants show an increase in the difference of slopes for $p p$ and $\bar{p} p$ scattering at the highest energies. This difference is smaller in the case of the slope at $q^{2}=0.05 \mathrm{GeV}^{2}$ and, as expected, the bulk of the experimental data are better described in this case. From both figures we also see that the effect of the experimental data at high values of the momentum transfer is an increase on the difference of the slopes for $p p$ and $\bar{p} p$ scattering. 

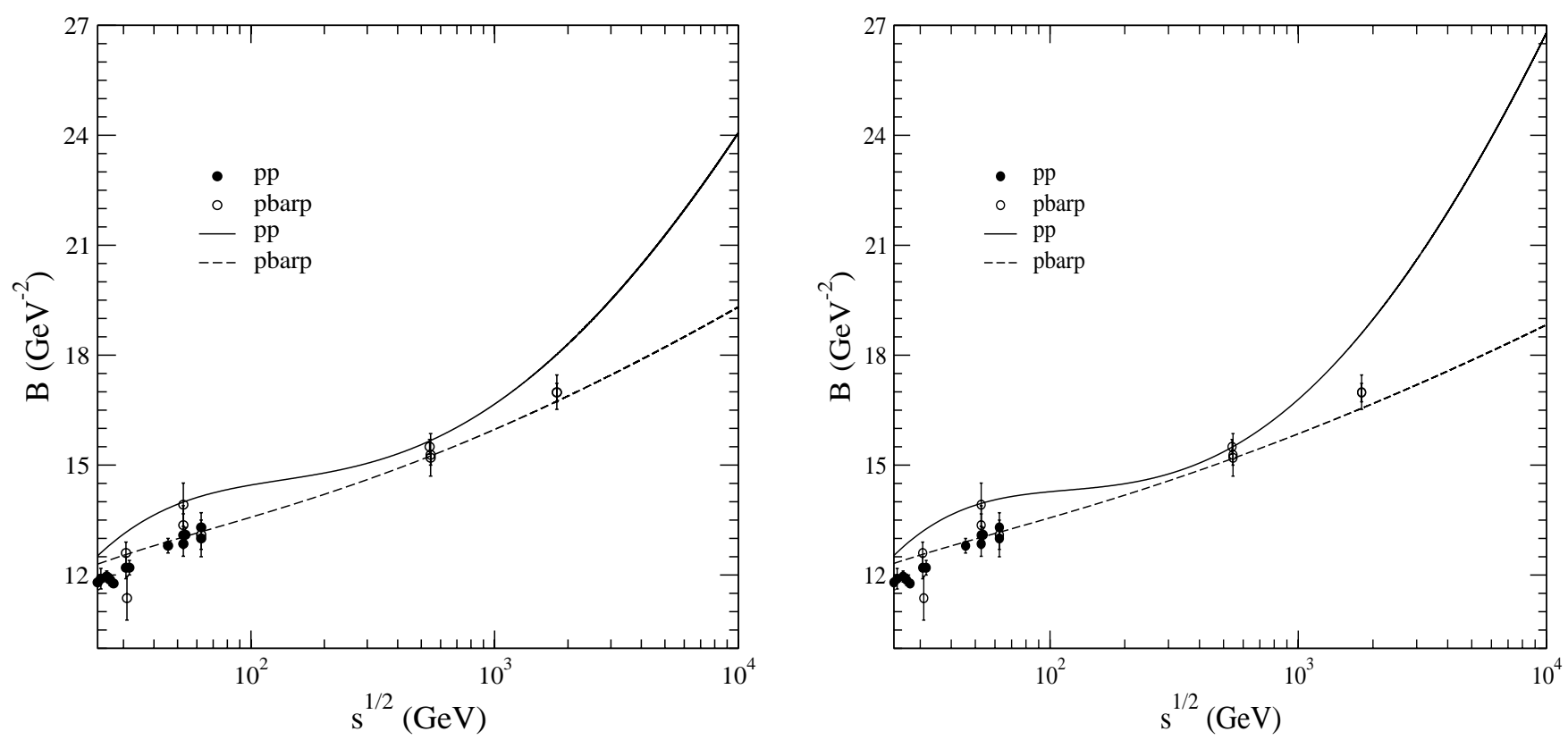

FIG. 2: Predictions for the slope at $q^{2}=0$ as function of the energy, from fits to the differential cross section data up to $q_{\mathrm{max}}^{2}=2 \mathrm{GeV}{ }^{2}$ (left) and $q_{\max }^{2}=14 \mathrm{GeV}^{2}$ (right).

\section{Acknowledgments}

We congratulate Professor Yogiro Hama for his 70th anniversary. For financial supports, S.D.C and M.J.M are thank- ful to BIG-UNICAMP and M.J.M. also to FAPESP (Contract No.04/10619-9).
[1] P.A.S. Carvalho, A.F. Martini, and M.J. Menon, Eur. Phys. J. C 39, 359 (2005).

[2] R.F. Ávila, S.D. Campos, M.J. Menon, and J. Montanha, Eur. Phys. J. C 47, 171 (2006)

[3] R.F. Ávila and M.J. Menon, Nucl. Phys. A 744, 249 (2004); J.
Fischer, P. Kolář, J. Math. Phys. 25, 2538 (1984); Phys. Lett. B 64, 45 (1976).

[4] Durham Database Group ( http://durpdg.dur.ac.uk/). 